Please quote as: Mrass, V.; Peters, C. \& Leimeister, J. M. (2017): Von Kunden für Kunden: Crowd Services als Erweiterung der Digital Customer Experience. In: HMD Praxis der Wirtschaftsinformatik, Ausgabe/Nummer: 5, Vol. 54, Erscheinungsjahr/Year: 2017. pp. 821-837. 


\section{Von Kunden für Kunden: Crowd Services als Erweiterung der Digital Customer Experience}

Volkmar Mrass ${ }^{1}$ • Christoph Peters ${ }^{2,1}$ • Jan Marco Leimeister 2, 1

Volkmar Mrass,

volkmar.mrass@uni-kassel.de

Tel.: +49 561 804-6066, ORCID: 0000-0003-4410-0022

Christoph Peters

christoph.peters@unisg.ch + christoph.peter@uni-kassel.de

Jan Marco Leimeister

janmarco.leimeister@unisg.ch + leimeister@uni-kassel.de

${ }^{1}$ Universität Kassel, Fachgebiet Wirtschaftsinformatik, Wissenschaftliches Zentrum für ITGestaltung, Pfannkuchstraße 1, $34121 \mathrm{Kassel}$, Deutschland

2 Universität St.Gallen, Institut für Wirtschaftsinformatik, Müller-Friedberg-Strasse 8, 9000 St.Gallen, Schweiz

This article is published in the journal „HMD Praxis der Wirtschaftsinformatik“, Vol. 54, No. 5/317, 2017 , p. 821-837. The final publication is available at https://link.springer.com/article/10.1365\%2Fs40702-017-0345-y. 


\title{
Von Kunden für Kunden: Crowd Services als Erweiterung der Digital Customer Experience
}

Zusammenfassung. Die „Plattform-Ökonomie“ verändert gegenwärtig die Art und Weise, wie Arbeit erbracht wird. Insbesondere Crowdworking-Plattformen haben in den letzten Jahren starken Zulauf erfahren und können von Unternehmen mittlerweile für eine Vielzahl von Tätigkeiten entlang ihrer Wertschöpfungskette genutzt werden. Ein Beispiel ist der Kundensupport, dem angesichts immer komplexerer Endprodukte große Bedeutung zukommt. Anhand der Crowdworking-Plattform Mila und ihres Kunden und 51-prozentigen Eigentümers Swisscom AG wird in diesem Beitrag aufgezeigt, wie Unternehmen die Crowd zur Erweiterung ihrer digitalen Schnittstelle zum Kunden einsetzen, ihren Service ausbauen und damit Mehrwert für alle Beteiligten generieren können. Die Crowdworking-Plattform Mila verbindet in einem neuartigen Ansatz das Paradigma der Crowd, insbesondere die Nutzung einer Vielzahl von Leistungserbringern, mit den Prinzipien der On-Demand und Sharing-Economy, vor allem dem Einbezug der Kunden als Produzenten von Dienstleistungen. Ähnlich wie bei prominenten plattformbasierten Beispielen der Sharing Economy wie Airbnb und Uber erfolgt auch hier die Anbahnung der Arbeit sowie die Schnittstelle zum Kunden auf digitalem Wege, während die Arbeit selbst meist direkt vor Ort beim Kunden erbracht wird. Basierend auf Experten-TiefenInterviews mit Verantwortlichen von Mila und Swisscom und weiteren Quellen analysieren wir in diesem Beitrag dieses neuartige Arbeitssystem inklusive des Zusammenspiels aller Beteiligten. Im Anschluss daran zeigen wir Vor- und Nachteile der Nutzung von Crowd Services auf und leiten konkrete Handlungsempfehlungen für Unternehmen für die Gestaltung der digitalen Schnittstelle zum Kunden ab.

Schlüsselwörter Crowdsourcing - Crowdworking-Plattformen - Crowd Services - Crowd Work • Digitale Kundenschnittstelle • On-Demand Ecomomy

\section{From customers for customers: Crowd services as an extension of the digital customer experience.}

\begin{abstract}
The "Platform Economy" is currently changing the way how work is performed. Particularly, crowdworking platforms have gained momentum during the last years. By now, they can be used by companies for a wide range of activities along their value chain. One example is the customer support that increasingly gains importance given the more and more complex end products. Based on the crowdworking platform Mila and its client and 51-percent owner Swisscom AG, we demonstrate how companies that use the crowd extend their digital interface to the customer and expand their services, generating an added value for all those involved. With its innovative approach, the crowdworking platform Mila connects the paradigm of the crowd, particularly the utilization of a variety of providers, with the principles of the On Demand- and Sharing Economy, primarily the inclusion of the customers as service "producers". Similarly to well-known platform-based examples of the Sharing Economy such as Airbnb and Uber, the initiation of work and the customer interface is shaped in a digital way, though the work itself is then usually performed directly on site at the customer's. Based on in-depth expert interviews with those responsible of Mila and Swisscom, we analyze this new kind of work system including the interplay of all participants. Subsequently, we show advantages and disadvantages of using crowd services and derive concrete recommendations of action for companies for the design of the digital customer interface.
\end{abstract}

Key Words Crowdsourcing • Crowdworking platforms $\bullet$ Crowd services $\bullet$ Crowd work $\bullet$ Digital customer interface $\bullet$ On demand ecomomy 


\section{Digitalisierung und Plattformökonomie}

Die Digitalisierung verändert die Art und Weise, wie Arbeit erbracht wird. Digitale Technologien sind wichtige Treiber dieser Transformation der Arbeitswelt sowie von Produktivität und Wachstum (Brynjolfsson und McAfee 2011). Eine große Anzahl innovativer, digitaler neuer Geschäftsmodelle der letzten Jahre basiert dabei auf dem Plattformprinzip, häufig ist auch von „Plattformökonomie“ die Rede. Eine „Spielart“ hiervon sind Crowdworking-Plattformen: Internet-basierte Plattformen, auf denen bezahlte Arbeiten mittels eines Aufrufs an eine größere Anzahl an potenziellen Bearbeitern („Crowd“) ausgeschrieben werden; diese Plattformen übernehmen als Intermediäre dabei die Abwicklung, Koordination und Steuerung. Damit verbunden ist ein Paradigmenwechsel: Arbeit wird den jeweiligen Bearbeitern nicht mehr zugewiesen, sondern diese wählen ihre Aufgaben selbst (Mrass et al. 2016).

Gerade für Ökonomien, die historisch eine starke industrielle Basis haben - wie das bei Deutschland als größter Volkswirtschaft Europas und viertgrößter Volkswirtschaft der Welt der Fall ist - gilt es, sich rechtzeitig auf diese Entwicklung einzustellen, um nicht weiter Boden gegenüber im Bereich plattformbasierter Geschäftsmodelle führenden Nationen wie den USA zu verlieren. Die Wettbewerbsfähigkeit ganzer Länder hängt davon ab, wie erfolgreich sie dieser Entwicklung begegnen können (Mrass et al. 2017b). Ausgehend von der Erkenntnis, dass auf technologischen Entwicklungen basierende neue Geschäftsmodelle langfristig nicht verhindert werden können - „,was digitalisiert werden kann, wird digitalisiert“ (Kollmann und Schmidt 2016, S. 70) - ist ein erster wichtiger Schritt hierzu eine intensive Untersuchung und ein tiefes Verständnis der Funktionsweise solcher Crowdworking-Plattformen. Die Anzahl der Unternehmen, die sich solcher Crowdworking-Plattformen zur Aufgabenerledigung bedienen, wächst, die Art der Arbeiten, die hierdurch erledigt werden können, ebenfalls (Mrass und Peters 2017). Gut ein Jahrzehnt nach der Einführung und Prägung des Begriffes durch Jeff Howe im Jahr 2006 kann heute eine Vielzahl an Tätigkeiten entlang der Wertschöpfungskette eines Unternehmens mit Hilfe der „Crowd“ abgewickelt werden (siehe auch Abbildung 1).

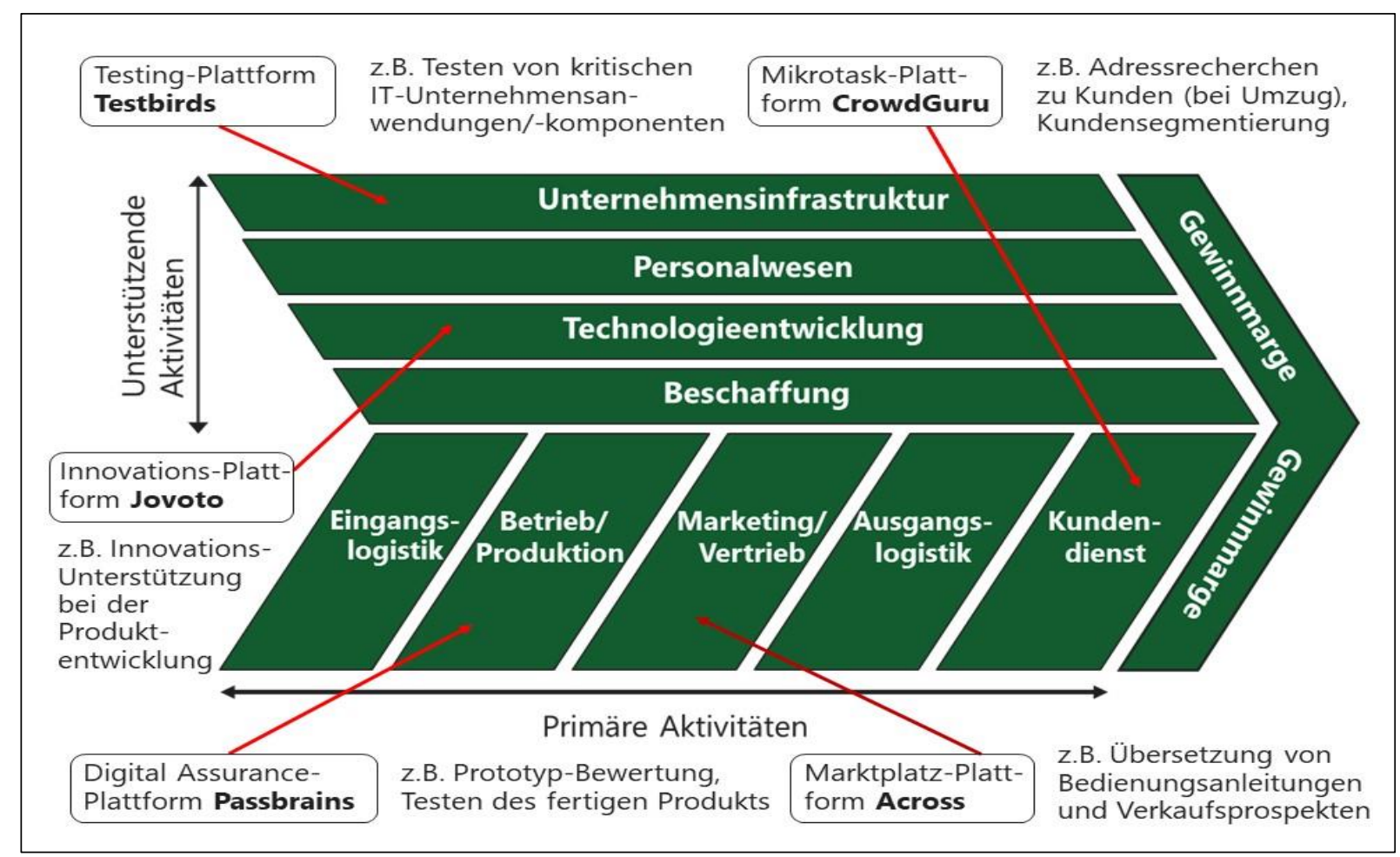

Abb. 1 Beispiele für den Einsatz von ausgewählten Crowdworking-Plattformen entlang der Porter'schen Wertschöpfungskette (Quelle: Eigene Darstellung in Anlehnung an Leimeister und Zogaj 2013, S. 10) 
Ein im deutschsprachigen Raum neuartiger Bereich in diesem Leistungsportfolio ist der der Crowd Services. Unternehmen bedienen sich dabei mittels Crowdworking-Plattformen als Intermediären einer Vielzahl an Bearbeitern, um Beratungs- und Vertriebsleistungen rund um ihre Produkte erbringen zu lassen. Dabei kommt es nicht zwingend zu einer „Kannibalisierung“ existierender Arbeit; es entstehen vielmehr neue Serviceangebote, die sich den Prinzipien der On-Demand und Sharing-Economy bedienen und für die betreffenden Unternehmen und ihre Kunden zusätzlichen Mehrwert schaffen. Ein interessantes Beispiel einer solchen Plattform ist Mila (www.mila.com) mit Sitz in Zürich sowie einem Deutschland-Standort in Berlin. Wir haben diese Plattform für diesen Beitrag unter anderem auf Grund der Tatsache ausgewählt, dass es sich hierbei um die einzige uns bekannte etablierte Service-/Vertriebs-Plattform dieser Art in Deutschland handelt (von einer Anfang 2017 neu gestarteten Plattform, die naturgemäß noch nicht über so lange Erfahrungen verfügen kann, mal abgesehen) und die Hauptzielgruppe der HMD-Leser aus dem deutschsprachigen Raum kommt. Bei den anderen im Feld vorhandenen Crowdworking-Plattform-Arten handelt es sich größtenteils um Plattformen, die Arbeit im „Backend" abwickeln und daher meist keine direkte Kommunikation mit den Endkunden der jeweiligen beauftragenden Unternehmen stattfindet (und damit auch keine Erweiterung der Digital Customer Experience dieser Unternehmen vorgenommen wird).

Das Unternehmen Mila bietet seinen Kunden - vornehmlich aus der Elektronik-, Energieversorgungs- und Telekommunikationsbranche - Services mit Schwerpunkt auf technischer Unterstützung durch seine Crowd, insbesondere seiner sogenannten „Friends“. Bei diesen handelt es sich um technikaffine Individuen, die sich mit Produkten des jeweiligen Kundenunternehmens gut auskennen, dieses im Rahmen einer Prüfung durch Mila nachgewiesen haben und zumeist in ihrer Freizeit eine Art „technische Nachbarschaftshilfe“ erbringen. Die Crowd der Mila Friends lässt sich nach der Zielgruppe des beauftragenden Unternehmens in weitere SubCommunities einteilen. Anhand einer solchen, den Swisscom Friends der Crowdworking-Plattform Mila, gehen wir in diesem Beitrag auf die Erweiterung der digitalen Kundenschnittstelle für Kunden von Unternehmen wie diesem führenden schweizer Telekommunikationsanbieter und die daraus ableitbaren Handlungsempfehlungen näher ein.

Unsere Einblicke und Erkenntnisse hierzu resultieren neben Gesprächen mit CrowdworkingExperten aus Unternehmen und Verbänden, Beiträgen aus öffentlich zugänglichen Quellen (u.a. Presseberichten und -mitteilungen), Brancheninformationen und dem Internet-Auftritt von Mila nicht zuletzt aus intensiven, ein- bis eineinhalbstündigen Tiefen-Interviews mit Verantwortlichen von Mila und Swisscom: So am 27. Januar 2017 mit Mila-CEO Christian Viatte und am 14. Februar 2017 mit dem Swisscom-Head of Customer Field Service (und damit Chef von rund 1.450 Mitarbeiterinnen und Mitarbeitern) Francesco Castelletti (letzterer ist zugleich auch eines der vier Mitglieder des Verwaltungsrates von Mila). Da es sich hierbei um ein neuartiges Phänomen handelt, lag unser zentrales Erkenntnisinteresse darin, explorativ (Yin 2014) zu untersuchen, wie Mila als Crowdworking-Plattform die Digital Customer Experience der Kunden von Swisscom erweitert, dieses Arbeitssystem (Alter 2013) inklusive der Rollenverteilung zwischen Mila und Swisscom zu analysieren und basierend darauf erste Handlungsempfehlungen unter anderem zur Integration dieser neuen Kundenschnittstelle für Unternehmen abzuleiten.

Die zentrale Forschungsfrage lautet dementsprechend: „Wie erweitert die Crowd ServicePlattform Mila die Digital Customer Experience der Privatkunden von Swisscom und welche ersten Handlungsempfehlungen lassen sich daraus für Unternehmen ableiten?" Dieser Beitrag hat folgenden weiteren Verlauf: Nach dieser Einführung (Abschnitt 1) wird die Crowd ServicePlattform Mila inklusive deren Zusammenspiel mit Swisscom vorgestellt (Abschnitt 2). Es folgt eine Analyse dieses Arbeitssystems (Abschnitt 3) und die Darstellung der Vor- und Nachteile solcher Crowd Services (Abschnitt 4). Schließlich werden konkrete Handlungsempfehlungen für Unternehmen abgeleitet und Schlussfolgerungen für die Zukunft gezogen (Abschnitt 5). 


\section{Mila: Kunden-Service durch die Crowd}

Im Jahr 2013 als Spin-off des Softwareherstellers Coresystems in der Schweiz gegründet und im Jahr 2014 auch in Deutschland gestartet, verfügt das junge Unternehmen Mila über eine rund 4.000-köpfige Crowd (in der Mehrzahl in der Freizeit tätige Friends, in der Schweiz aber auch hauptberuflich tätige Profis), die etwa 4.000 Serviceaufträge pro Monat bearbeitet und in 150 Städten aktiv ist. Das Unternehmen selbst hat 26 eigene Mitarbeiter, die sich auf die Standorte Zürich (Sitz) und Berlin verteilen. Ende 2015 übernahm das schweizer Telekommunikationsunternehmen Swisscom AG die Mehrheit an Mila. Mila vermittelt technik-affine Mitglieder der Crowd an Endkunden, die bei der Einrichtung ihrer Technik selbst nicht weiterkommen, ein technisches Problem haben oder schlichtweg nicht die notwendige Zeit aufbringen wollen, um sich damit zu beschäftigen. Der persönliche Kontakt zwischen diesen Friends von Mila und den Kunden der Plattform beziehungsweise der Unternehmen wird online vermittelt, dabei wird auf zeitliche Flexibilität hohen Wert gelegt. Die Reaktionszeit ist gering, gemäß Mila werden über 40 Prozent der Aufträge innerhalb einer Minute angenommen. Innerhalb von 10 Minuten sind es 70 Prozent, innerhalb einer Stunde über 90 Prozent und nach drei Stunden sind 98 Prozent der Aufträge verteilt. Eine Service-Anfrage eines Kunden wird an die geografisch relevante Crowd der Mila Friends in der Nähe (in der Regel in einem Umkreis von 30 Kilometern) ausgeschrieben, was eine zeitnahe Anreise und damit verbunden geringere Kosten ermöglicht. Derjenige Crowd Worker (Friend), der den Job zuerst annimmt, führt ihn auch aus. Neben dem Zugang per PC (siehe Abbildung 2) gibt es seit Februar 2017 auch eine neue App für Smartphones mit integriertem Service-Bot und On-Demand-Funktion, die eine Buchung von Services zu einem vom Kunden bestimmten Wunschtermin inklusive der Möglichkeit, „,sofort“ (i.d.R. 10 bis 60 Minuten) technische Unterstützung zu erhalten, beinhaltet (siehe Abbildung 3).

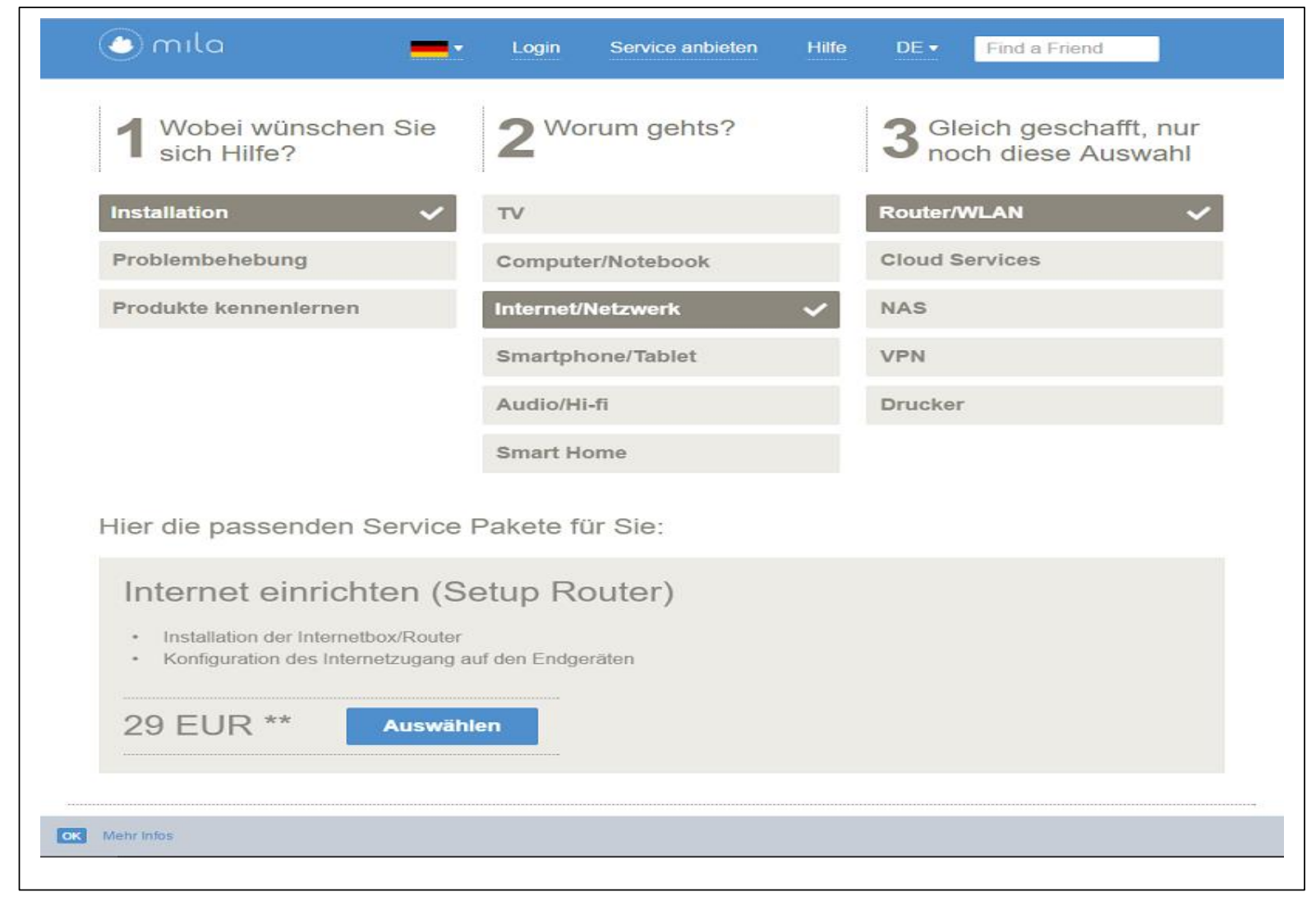

Abb. 2 Digitale Kundenschnittstelle für Mila-Kunden über das Internet mit Ansicht der auswählbaren Services (Quelle: https://www.mila.com/milade, abgerufen am 06.03.2017) 


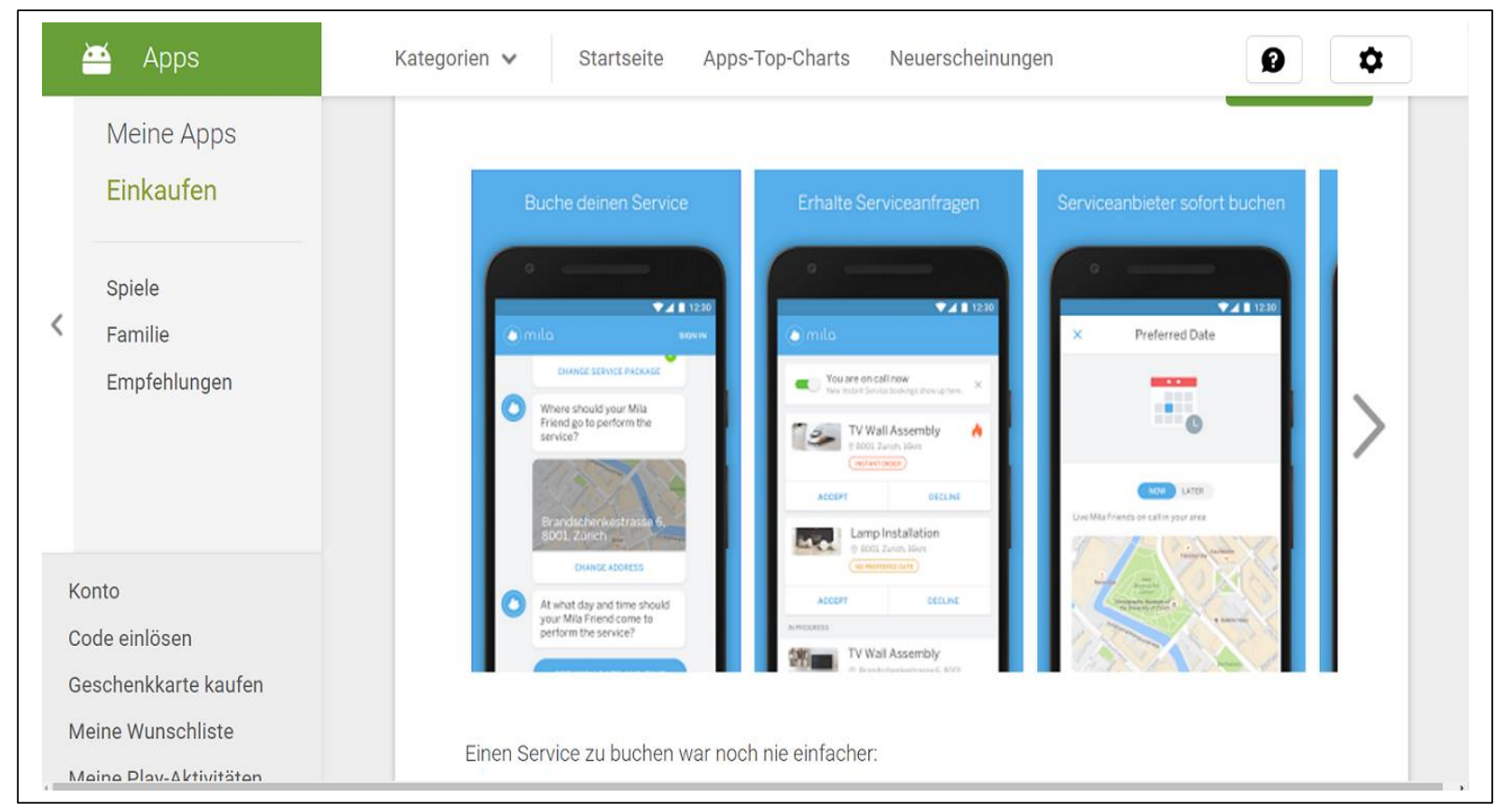

Abb. 3 Digitale Kundenschnittstelle für Mila-Kunden über neue App für Mobilfunkgeräte mit Orts-Ansicht (Quelle: https://play.google.com/store/apps/details?id=com.mila.app, abgerufen am 06.03.2017)

Der Schwerpunkt der Services liegt im Bereich der Installation, der Problembehebung oder Produkterläuterung zu TV-Geräten, Computern und Notebooks, Internet/Netzwerken, mobilen Endgeräten, Audio/Hifi oder SmartHome-Anwendungen. Mila nennt für den jeweiligen Service Orientierungs-Preise, die Vereinbarung des endgültigen Preises erfolgt jedoch direkt zwischen dem Mila Friend und dem Kunden und kann davon abweichen. Weitere Leistungen sind in Abstimmung zwischen den beiden Vertragsparteien ebenfalls möglich. Die Buchung, Abwicklung und Steuerung der jeweiligen Services erfolgt über die Mila-Plattform als Intermediär und Vermittler zwischen der Crowd und den Kunden. Der geografische Schwerpunkt der erbrachten Services liegt gegenwärtig in der Schweiz und Deutschland, wobei aktuell ein Marktlaunch in Polen, Österreich und Frankreich erfolgt und in Australien ein Pilotbetrieb stattfindet.

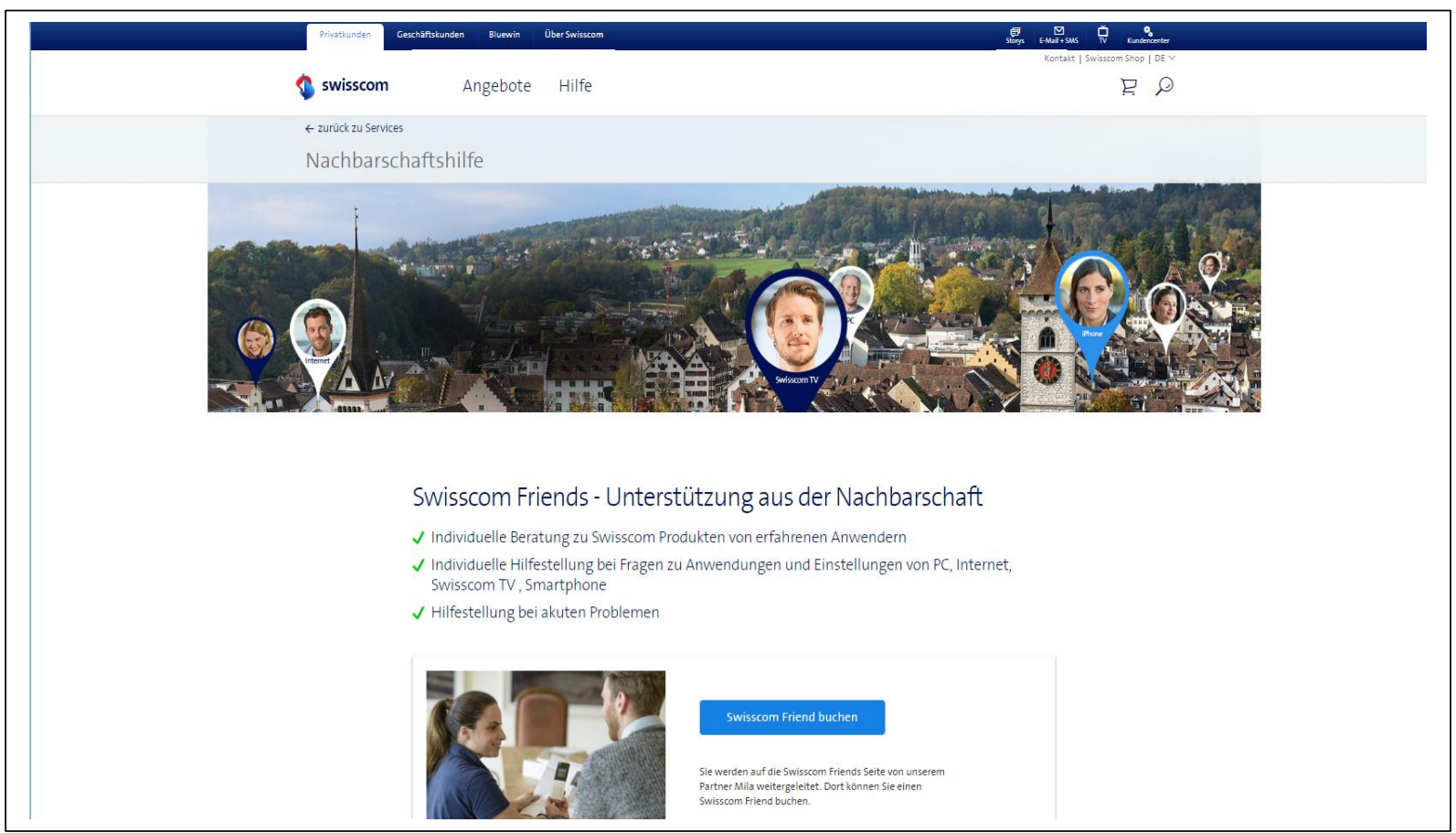

Abb. 4 Digitale Kundenschnittstelle für Swisscom-Privatkunden über den Internet-Auftritt von Swisscom (Quelle: https://www.swisscom.ch/de/privatkunden/services/friends.html, abgerufen am 10.03.2017) 
Swisscom nutzt Mila als zusätzlichen Kanal zum eigenen Kundendienst sowie POS-Personal für die Erbringung von Services rund um die Produkte. Je nach Anlass und Ziel wird dabei zwischen den jeweiligen Vertriebskanälen der passende Service empfohlen: Erweist sich beispielsweise ein Problem vor Ort als zu komplex und nicht durch Mila Friends zu beheben, wird auf den regulären Swisscom Kundendienst verwiesen. Umgekehrt sind die Swisscom Friends in das Service-Angebot des Unternehmens als ein Zugangskanal integriert (siehe Abbildung 4).

\section{Erweiterung der Digital Customer Experience}

Am Beispiel der Crowdworking-Plattform Mila und ihres Kunden (und zugleich 51-prozentigen Eigentümers) Swisscom lässt sich gut aufzeigen, wie Unternehmen heute das Prinzip der „Wisdom of Crowds“ (Surowiecki 2004) nutzen können, um ihren Service auszubauen und ihre Digital Customer Experience zu erweitern. Und dabei zugleich dem Trend der On-DemandEconomy, die je nach Bedarf kurzfristig zu erbringende Services erfordert, sowie der SharingEconomy, die Kunden zugleich zu Konsumenten und Produzenten (,Prosumenten“) macht und ungenutzte Ressourcen teilt, zu begegnen. Ähnlich wie ein Fahrer bei Uber sein Auto bereitstellt und sich zwecks Abholung zum Kunden begibt, stellt ein Mila Friend Ressourcen wie seinen privaten Laptop zur Verfügung (z.B. zwecks Analyse oder Vornahme von technischen Konfigurationen) und begibt sich ebenfalls zum Kunden, um dort seine Services zu erbringen. Für die Kunden von Swisscom erfolgt durch die Bereitstellung der in Abschnitt 2 vorgestellten Beauftragungsmöglichkeiten über die Internet-Auftritte von Mila und Swisscom sowie über die Mobile App eine substanzielle Erweiterung der Digital Customer Experience. Sie erhalten damit weitere Kommunikationskanäle und digitale Zugangsmöglichkeiten zum Unternehmen. Neben den bestehenden digitalen Produkten von Swisscom selbst wird damit auch die Anzahl der digitalen Services für die Kunden erhöht. Das Unternehmen Swisscom gewinnt auf Grund dieses Angebotes auch insbesondere junge, technik-affine Kundengruppen hinzu, die die Abwicklung von Services über digitale Kanäle auch bereits aus anderen Lebensbereichen kennen. Zugleich bedeutet dieses erweiterte digitale Angebot auch für die Teil-Kundengruppe der Swisscom Friends eine Erweiterung ihrer Digital Customer Experience, da diese nun neben ihrer Rolle als Konsumenten der Produkte und Services von Swisscom gleichzeitig auch zu Mitwirkenden am Leistungserstellungsprozess werden. Letzterer wird für die Swisscom Friends ebenfalls digital über die Crowdworking-Plattform Mila gesteuert und koordiniert.

Auf Basis einer Analyse des Arbeitssystems („Work System“) der Crowdworking-Plattform Mila mittels des Work System Frameworks von Alter (2013, S. 78) ermitteln wir im Folgenden Vor- und Nachteile der Nutzung solcher Crowd Services. Wir wählen dieses Framework und die dahinter liegende Work System Theorie von Alter (2013) als Instrumente, da beide eine sehr gute Basis für die Analyse des Zusammenspiels zwischen Teilnehmenden (vor allem Mila Friends, teilweise aber auch die Kunden), Information (beispielsweise das Wissen rund um Swisscom-Produkte) und Technologien (beispielsweise die Mila Mobile App) zur Durchführung der Prozesse und Aktivitäten (beispielsweise Installation, Problembehebung und Produktberatung) bieten. Das Work System Framework ermöglicht insbesondere auch gut die Analyse von sozio-technischen Systemen wie demjenigen, in das Mila und Swisscom eingebunden sind.

Um die Analyse zu strukturieren, führen wir diese und die Ermittlung der Vor- und Nachteile entlang der internen Kern-Elemente des Work System Frameworks Prozesse und Aktivitäten, Teilnehmende, Information und Technologien durch. Sowie zusätzlich auch entlang der in diesem Fall ebenfalls innerhalb des Work Systems befindlichen Kunden (da Kunden hier gleichzeitig auch Teilnehmende sind, die bei der Service-Erbringung mitwirken und die Services erst im Zusammenspiel zwischen Crowd und Kunden Gestalt annehmen) und Produkte \& Services (siehe Abbildung 5). Basierend auf den ermittelten Vor- und Nachteilen leiten wir im Anschluss daran konkrete Handlungsempfehlungen für Unternehmen ab. 


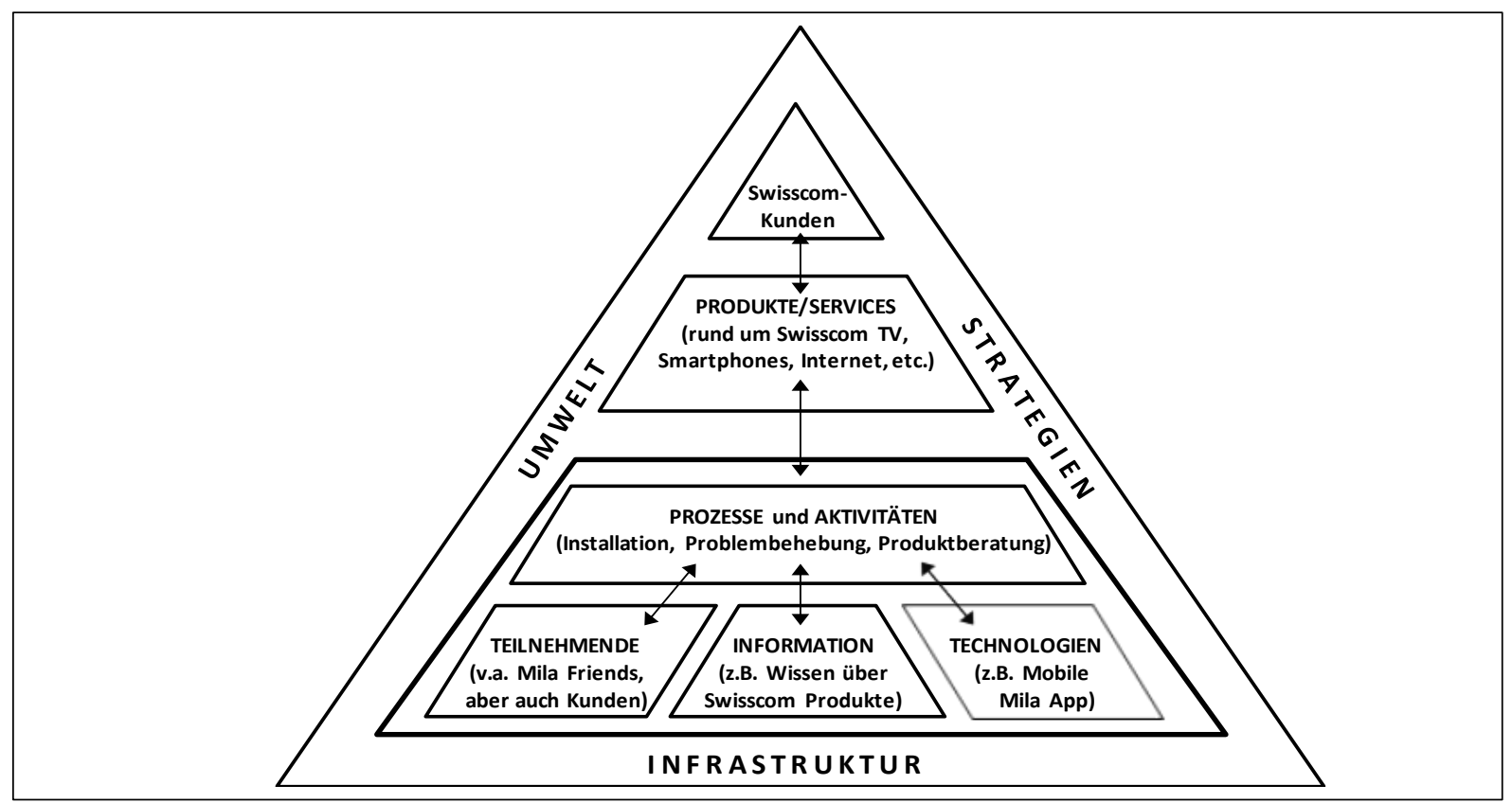

Abb. 5 Darstellung des Arbeitssystems/Work Systems von Mila und dem Kunden Swisscom in Anlehnung an Alter's Work System Frameworks (Quelle: eigene Darstellung in Anlehnung an Alter 2013, S. 78)

\section{Vor- und Nachteile der Nutzung von Crowd Services}

Am Beispiel Mila lassen sich Vor- und Nachteile der Nutzung von Crowd Services für Unternehmen ableiten, die hier nun stichpunktartig dargestellt und entlang der Elemente des Arbeitssystems/Work System Frameworks erläutert werden. In die Ermittlung dieser Vor- und Nachteile sowie der in Abschnitt 5 daraus abgeleiteten Handlungsempfehlungen für Unternehmen sind neben Erkenntnissen aus dem Case Mila \& Swisscom auch Erkenntnisse und Erfahrungen, welche die Autoren dieses Beitrages im Rahmen ihrer allgemeinen weiteren Forschung zu Crowdworking-Plattformen, insbesondere in Untersuchungen von insgesamt 14 solcher Plattformen in Deutschland gewonnen haben (siehe Tabelle 1), mit eingeflossen:

\begin{tabular}{|l|l|l|l|l|}
\hline Plattform & Sitz/Standort & Art der Plattform & Haupt-Interview-Partner & Interview-Datum \\
\hline Across & Karlsbad & Marktplatz & Christian Weih (CSO) & 7. Juni 2016 \\
\hline CrowdGuru & Berlin & Mikrotask & Hans Speidel (CEO) & 6. Juli 2016 \\
\hline Jovoto & Berlin & Design/Innovation & Bastian Unterberg (CEO) & 19.7.2016+1.6.2017 \\
\hline Testbirds & München & Testing & Markus Steinhauser (COO) & 21. Juli 2016 \\
\hline Passbrains & Hamburg & Digital Assurance & Dieter Speidel (CEO) & 9. August 2016 \\
\hline Clickworker & Essen & Mikrotask & Ines Maione (PR Manager) & 26. September 2016 \\
\hline Content.de & Herford & Content/Text & Dr. Arne-Chr. Sigge (CEO) & 27. September 2016 \\
\hline Innosabi & München & Innovation & Jan Fischer/GF & 28. September 2016 \\
\hline Twago & Berlin & Marktplatz & Thomas Jajeh/CEO & 28. September 2016 \\
\hline TestIO & Berlin & Testing & Philipp Soffer/CEO & 30. September 2016 \\
\hline Mila & Berlin & Vertrieb & Christian Viatte/CEO & 27. Januar 2017 \\
\hline Phantominds & Hamburg & Innovation & Dr. Mirko Bendig/GF & 21.2.+13.6.2017 \\
\hline Hyve & München & Innovations & Markus Rieger/GF & 31. Mai 2017 \\
\hline Local Motors & Berlin & Engineering & Samuel Buschhorn/Manager & 11. Juli 2017 \\
\hline
\end{tabular}

Tab. 1 Übersicht der im Rahmen der weiteren Forschung der Autoren zu Crowdworking-Plattformen näher untersuchten insgesamt 14 Crowdworking-Plattformen in Deutschland und der jeweiligen Haupt-Interview-Partner 
Kunden. An der Spitze des Work System Frameworks und im Mittelpunkt der Anstrengungen von Unternehmen stehen die Kunden. Die Kunden von Swisscom erhalten auf Grund der groBen Anzahl an möglichen Bearbeitern aus der Mila-Crowd und der Tatsache, dass diese auch abends und an Wochenenden zur Verfügung stehen, kurzfristig Unterstützung. In der Regel wird ein Auftrag - siehe Abschnitt 2 - spätestens innerhalb von 3 Stunden angenommen. Mittels der Smartphone-App besteht zudem die Möglichkeit der Buchung von kurzfristigen Services, bei denen eine technische Unterstützung innerhalb von 10 bis 60 Minuten erfolgt. Ein wesentlicher Vorteil für die Kunden im Vergleich zu von internen Mitarbeitern erbrachten Services ist also die Geschwindigkeit der Service-Erbringung. Swisscom kann seinen Kunden mittels der Crowd Services auch zu Zeiten anbieten, die außerhalb der regulären Kundendienstzeiten liegen. Aus der Tatsache, dass die „Mila-Friends“ in der Regel aus kurzer Entfernung anreisen und diese Services meist nebenher in ihrer Freizeit erbringen, resultiert ein weiterer Vorteil für die Kunden von Swisscom: Im Vergleich zu den durch interne Mitarbeitern von Swisscom erbrachten Services sind die Kosten in diesem Fall für sie deutlich geringer.

Neben diesen Vorteilen, die Unternehmen ihren Kunden bieten können, beinhaltet der Einsatz der Crowd auch Nachteile: Die Einheitlichkeit des Auftritts beim Kunden - Stichwort „Corporate Identity" - lässt sich für Unternehmen mit eigenen Beschäftigten in der Regel besser gewährleisten als mit einer relativ heterogeneren externen Crowd. Interne Mitarbeiter sind zudem mit der Kultur und Vorgehensweise eines Unternehmens besser vertraut als externe Crowd Worker und können damit vor Ort beim Kunden stärker im Sinne des Unternehmens handeln.

Produkte \& Services. Zentrales Ergebnis der Anstrengungen und Tätigkeiten von Unternehmen sind Produkte und Services. Swisscom augmentiert mittels der Mila Crowd, die über ein hohes technisches Wissen verfügt, die bisherigen Services rund um Produkte wie Internet, Smartphones, Tablets, TV oder Wearables. Das Unternehmen verfügt mittels Mila Friends über eine weitere digitale Kundenschnittstelle und einen zusätzlichen Kanal, über den Services rund um diese Produkte beauftragt werden können. Wie oben bereits geschildert, sind die Kosten für diese Services geringer als bei der Leistung durch interne Mitarbeiter. Damit ist dieses Angebot nach Angabe der Verantwortlichen von Mila und Swisscom auch für Kunden attraktiv, die nicht bereit sind, für diese Art von Services die regulär anfallenden Preise zu bezahlen. Gerade für Tätigkeiten, die - durch die hauptamtlichen Beschäftigten eines Unternehmens erbracht - relativ aufwändig und teuer wären und daher von Kunden eher gemieden werden, wird eine bestehende Lücke im Service-Angebot geschlossen. Swisscom als Unternehmen mit Wurzeln in der Schweiz kann zudem durch Einbindung der Crowd auch Services für seine Produkte in Ländern anbieten, in denen es nicht oder nur in sehr geringem Umfang vertreten ist und damit die Präsenz seiner Marke erhöhen. Das Angebot einer neuen Kundenschnittstelle, über die ServiceAufträge rund um die Produkte getätigt werden können, und die geografische Ausweitung des Vertriebsgebietes sind also weitere Vorteile für Unternehmen bei der Nutzung dieses Modells.

Auf der anderen Seite werden Crowd Worker/Friends, obwohl keine Angestellten des Unternehmens, sondern selbständig tätig, bei der Erbringung der Services dennoch oft als Unternehmens-Repräsentanten wahrgenommen. Das kann sich insbesondere bei Fehlern bei der Erbringung der Services, mangelnder Kompetenz oder Unfreundlichkeit der Crowd Worker negativ auf die Kundenzufriedenheit und damit die Marke und das Unternehmen auswirken. Das Unternehmen hat hierauf jedoch gleichzeitig nur begrenzte Einfluss- und Steuerungsmöglichkeiten. Die Erbringung der Services durch externe Crowds kann außerdem zu einem Verlust an potenziellen Umsatz für das jeweilige Unternehmen führen. Das gilt insbesondere, wenn es sich nicht um Services handelt, die weitgehend komplementär sind, weil es zu den regulären Preisen und Konditionen des Kundendienstes des jeweiligen Unternehmens keinen Bedarf/Markt gäbe. Wenn es sich also um Services handelt, die der Kunde auch von internen Mitarbeitern des Unternehmens beziehen könnte, entgeht dem Unternehmen ein Teil des potenziellen Umsatzes. 
Prozesse \& Aktivitäten. Die Erbringung der Leistungen eines Unternehmens ist mit Prozessen und Aktivitäten verbunden, für deren zustande kommen wiederum Teilnehmende, Informationen und Technologien notwendig sind. Im Kontext dieser Prozesse und Aktivitäten ist bezogen auf Mila festzustellen, dass ein vermittelter „Friend“ auf Grund der durch die Plattform gesteuerten Auswahl nach räumlicher Nähe zum Kunden lange Anfahrtswege vermeidet. Dies erhöht die Effizienz und Schnelligkeit der Leistungserbringung. Gleichzeitig erfolgt eine passgenauere Konzentration auf die jeweiligen lokalen Gegebenheiten. Daraus lässt sich der Vorteil ableiten, dass Unternehmen ihre Prozesse und Aktivitäten zur Erbringung von Services durch Einbindung solcher Crowds beschleunigen, effizienter gestalten und zielgerichteter auf die Erfordernisse des Marktes und lokale Erfordernisse ausrichten können.

Andererseits sind die Kontinuität und Verfügbarkeit einer gut qualifizierten Crowd für die Abwicklung der Prozesse und Aktivitäten essenziell. Crowdworking-Plattformen als Intermediäre können diese aber nicht garantieren. In Zeiten, in denen die Crowd beispielsweise durch den Einsatz für andere Unternehmen stark gefordert ist, mag die Arbeit für das eigene Unternehmen leiden. Auch der umgekehrte Fall, dass sich Mitglieder der Crowd abwenden, da nicht genügend Aufträge eingehen, und ihre Arbeitskraft lieber anderen Crowdworking-Plattformen und den sie beauftragenden Unternehmen zur Verfügung stellen, ist denkbar.

Teilnehmende. Bei Teilnehmenden handelt es sich um Personen (oder auch Maschinen), welche die Arbeit innerhalb eines Arbeitssystems erbringen. Neben den Kunden von Swisscom, die an der Erbringung der Services mitwirken und daher zu den Teilnehmenden gehören, sind das hier insbesondere die Mila Friends. Diese sind selbständig tätig und nicht bei Mila oder Swisscom angestellt. Für die Mila Friends fallen also auf Seiten von Mila und Swisscom keine Löhne oder Sozialabgaben an, da deren Bezahlung (und damit indirekt auch die darin enthaltene Provision für die Vermittlungsplattform) von den Kunden übernommen wird. Daraus lässt sich der Vorteil für Unternehmen ableiten, durch Einbindung einer Crowd über eine Vermittlungsplattform Services rund um ihre Produkte anbieten zu können, für die keine Bezahlung in Form von Löhnen oder Sozialabgaben anfällt, da diese direkt von den Kunden übernommen wird.

Gleichzeitig kann dem Unternehmen dabei - wie bereits dargestellt - auch potenzieller Umsatz entgehen. Hinzu kommt, dass das Kompetenzniveau solcher Individuen - obwohl dieses durch die Crowdworking-Plattform im Vorfeld geprüft wird - in der Regel dennoch deutlich heterogener als dasjenige der nach einheitlichen Kriterien und Standards geschulten eigenen Beschäftigten ist. Der Einfluss auf und die Kontrolle über eigene Beschäftigte ist nicht zuletzt auf Grund des Direktionsrechts deutlich höher, ebenso die Sanktionierungs-Möglichkeiten bei Fehlverhalten. Gleichzeitig kann der Einsatz Externer zu Verunsicherung bei den internen Mitarbeitern bis hin zu im schlimmsten Fall Jobverlustängsten/inneren Kündigungen führen.

Information. Für das Erstellen von Produkten oder die Erbringung von Services werden Informationen genutzt. Im Fall der Swisscom-Friends von Mila werden beispielsweise gute Kenntnisse der Produkte benötigt. Die Swisscom Friends von Mila sind meist selbst Kunden von Swisscom, haben sich also oft auch privat freiwillig für das jeweilige Produkt entschieden. Daraus lässt sich für Unternehmen folgender Vorteil ableiten: Der Einsatz solcher Crowds, die die Produkte des Unternehmens selbst nutzen, ohne dafür beispielsweise auf Grund der Tätigkeit als interne Mitarbeiter eine gewisse „Verpflichtung“ zu haben, bringt ein hohes Maß an Glaubwürdigkeit bei der Kommunikation von Informationen/Empfehlungen für das Unternehmen mit sich. Die Mitglieder der Crowd sind damit gute und sehr glaubwürdige Markenbotschafter.

Trotz dieses hohen Maßes an Glaubwürdigkeit verfügen externe Crowd Worker im Durchschnitt nicht über die gleichen Informationsquellen wie interne Mitarbeiter und damit oft auch nicht über gleich gute Kenntnisse der Produkte eines Unternehmens. Dieses kann sich negativ auf die Qualität der durch diese Crowd Worker im Auftrag des Unternehmens erbrachten Services und damit auf den Geschäftserfolg des Unternehmens auswirken. 
Technologien. In der heutigen Arbeitswelt bilden Technologien meist die Basis für die Abwicklung von Prozessen und Aktivitäten. In diesem Fall sind das beispielsweise die InternetAuftritte von Swisscom und Mila, über die Kunden Leistungen beauftragen können, oder die mobile Mila-App. Gemäß Einschätzung von Swisscom wird die Bedeutung digitaler Kanäle wie der über die Plattform Mila langfristig im Vergleich zu Call Centern oder physischen POSKanälen vor Ort deutlich zunehmen. Mittels Mila kann Swisscom zudem eine Beschleunigung der Bearbeitung von Anfragen erreichen, da nun deutlich mehr potenzielle Bearbeiter zur Verfügung stehen und eine effiziente zentrale Steuerung der durch die Crowd erbrachten Services stattfindet. Daraus lässt sich der Vorteil ableiten, dass Unternehmen durch die Nutzung von auf Basis von Plattform-Technologien erbrachten Services Vorteile im Bereich der Beschleunigung der Abwicklung und der Effizienz der Steuerung des Arbeitssystems erzielen können.

Je nach Kundengruppe, Produkt und Unternehmen kann eine Abwicklung und Steuerung der Services durch solche Technologien aber auch nur die zweitbeste Lösung sein. So ist beispielsweise gerade bei technischen Unterstützungs-Leistungen wie bei Mila und Swisscom davon auszugehen, dass diese auch von vielen Kunden beauftragt werden, die weniger technikaffin sind (z.B. ältere Kunden). Gerade für diese Zielgruppe ist fraglich, ob beispielsweise die Beauftragung von Services per App der beste Zugangskanal ist.

Die Abbildungen 6 und 7 zeigen nochmals die wesentlichsten Vor- und Nachteile auf.

\begin{tabular}{|c|c|c|c|c|c|}
\hline Kunden & $\begin{array}{c}\text { Produkte \& } \\
\text { Services }\end{array}$ & $\begin{array}{l}\text { Prozesse } \& \\
\text { Aktivitäten }\end{array}$ & $\begin{array}{c}\text { Teil- } \\
\text { nehmende }\end{array}$ & $\begin{array}{l}\text { Infor- } \\
\text { mation }\end{array}$ & $\begin{array}{l}\text { Techno- } \\
\text { logien }\end{array}$ \\
\hline $\begin{array}{l}\text {-Hohe } \\
\text { Geschwindig } \\
\text { keit bei der } \\
\text { Erbringung } \\
\text { der Services } \\
\text { - Geringere } \\
\text { Kosten für } \\
\text { die Kunden } \\
\text {-Services } \\
\text { auch abends } \\
\text { und an } \\
\text { Wochen- } \\
\text { enden }\end{array}$ & $\begin{array}{l}\text {-Augmentier } \\
\text { ung des } \\
\text { Service- } \\
\text { Angebotes } \\
\text { - Neue } \\
\text { digitale } \\
\text { Kunden- } \\
\text { schnittstelle } \\
\text {-Geogra- } \\
\text { fische } \\
\text { Ausweitung } \\
\text { des Produkt- } \\
\text { Vertriebs- } \\
\text { gebietes }\end{array}$ & $\begin{array}{l}\text { - Gestiegene } \\
\text { Effizienz und } \\
\text { Schnelligkeit } \\
\text { der } \\
\text { Leistungs- } \\
\text { erbringung } \\
\text {-Bessere } \\
\text { Ausrichtung } \\
\text { der Prozesse } \\
\text { und Aktivi- } \\
\text { täten auf die } \\
\text { Erforder- } \\
\text { nisse des } \\
\text { Marktes }\end{array}$ & $\begin{array}{l}\text { •Keine Löhne } \\
\text { oder Sozial- } \\
\text { abgaben des } \\
\text { Unter- } \\
\text { nehmens für } \\
\text { die Crowd } \\
\text { Worker fällig } \\
\text { (Bezahlung } \\
\text { erfolgt } \\
\text { direkt durch } \\
\text { die Kunden) }\end{array}$ & $\begin{array}{l}\text { •Hohe } \\
\text { Glaubwürdig } \\
\text { keit bei der } \\
\text { Kommuni- } \\
\text { kation von } \\
\text { Information } \\
\text { en/Produkt- } \\
\text { Empfeh- } \\
\text { lungen } \\
\text {-Gute } \\
\text { "Marken- } \\
\text { botschafter" }\end{array}$ & $\begin{array}{l}\text {-Erhöhung } \\
\text { der Effizienz } \\
\text { der Steuer- } \\
\text { ung des } \\
\text { gesamten } \\
\text { Arbeits- } \\
\text { systems }\end{array}$ \\
\hline
\end{tabular}

Abb. 6 Vorteile der Nutzung von Crowd Services durch Unternehmen (Quelle: eigene Darstellung)

\begin{tabular}{|c|c|c|c|c|c|}
\hline Kunden & $\begin{array}{c}\text { Produkte } \boldsymbol{\&} \\
\text { Services }\end{array}$ & $\begin{array}{l}\text { Prozesse \& } \\
\text { Aktivitäten }\end{array}$ & $\begin{array}{c}\text { Teil- } \\
\text { nehmende }\end{array}$ & Information & $\begin{array}{l}\text { Techno- } \\
\text { logien }\end{array}$ \\
\hline $\begin{array}{l}\text {-Corporate } \\
\text { Identity" } \\
\text { und Einheit- } \\
\text { lichkeit des } \\
\text { Auftritts } \\
\text { beim } \\
\text { Kunden } \\
\text { schwerer zu } \\
\text { gewähr- } \\
\text { leisten }\end{array}$ & $\begin{array}{l}\text { - Fehler bei } \\
\text { Service- } \\
\text { Erbringung } \\
\text { wirken sich } \\
\text { negativ auf } \\
\text { Marke aus } \\
\text { - Gleichzeitig } \\
\text { begrenzte } \\
\text { Einfluss- und } \\
\text { Steuerungs- } \\
\text { möglich- } \\
\text { keiten }\end{array}$ & $\begin{array}{l}\text {-Kontinuität } \\
\text { bei Abwick- } \\
\text { lung der } \\
\text { Prozesse + } \\
\text { Aktivitäten } \\
\text { ist schwerer } \\
\text { zu gewähr- } \\
\text { leisten } \\
\text {-u.a. wegen } \\
\text { geringerer } \\
\text { Verfügbar- } \\
\text { keit }\end{array}$ & $\begin{array}{l}\text { - Hetero- } \\
\text { genes } \\
\text { Kompetenz- } \\
\text { niveau } \\
\text { - Geringere } \\
\text { Sanktions- } \\
\text { möglich- } \\
\text { keiten } \\
\text {-Verunsicher- } \\
\text { ung der } \\
\text { internen } \\
\text { Mitarbeiter }\end{array}$ & $\begin{array}{l}\text { - Friends } \\
\text { verfügen } \\
\text { nicht über } \\
\text { gleiche } \\
\text { Information } \\
\text { en und } \\
\text { Produkt- } \\
\text { kenntnisse } \\
\text {-Das kann } \\
\text { sich negativ } \\
\text { auf Qualität } \\
\text { auswirken }\end{array}$ & $\begin{array}{l}\text {-Bei einigen } \\
\text { Kunden } \\
\text { kann Ein- } \\
\text { satz von } \\
\text { Techno- } \\
\text { logien nach- } \\
\text { teilig sein } \\
\text {-Beispiel: } \\
\text { Nutzung von } \\
\text { Apps durch } \\
\text { ältere } \\
\text { Kunden }\end{array}$ \\
\hline
\end{tabular}

Abb. 7 Nachteile der Nutzung von Crowd Services durch Unternehmen (Quelle: eigene Darstellung) 


\section{Fazit: Fünf Handlungsempfehlungen für Unternehmen}

Wie in diesem Beitrag gezeigt wurde, liefert die Exploration und Analyse der im deutschsprachigen Raum neuartigen Crowd Services-Plattform Mila und des Zusammenspieles mit deren Kunden und Eigentümer Swisscom AG interessante Einblicke, die die Vor- und Nachteile dieses Modells sichtbar machen. In Kombination mit Erkenntnissen aus der Untersuchung weiterer Crowdworking-Plattformen durch die Autoren dieses Beitrages im Rahmen ihrer allgemeinen Forschung zu Crowdworking-Plattformen (siehe Tabelle 1) wurden weitere mögliche Vor- und Nachteile für Unternehmen skizziert. Auf Basis dieser Vor- und Nachteile werden nun im Folgenden fünf aus Sicht der Autoren besonders essenzielle, konkrete Handlungsempfehlungen für Unternehmen, die Crowdworking-Plattform für die Vermittlung von Crowd Services nutzen möchten, abgeleitet. Diese Empfehlungen gelten grundsätzlich für Unternehmen verschiedenster Branchen, wenngleich sich einige Branchen wie Finanzdienstleistungen, Energieversorgung oder Information- und Telekommunikation oder Tourismus besonders gut hierfür eignen. Die Empfehlungen gelten auch bei der Erbringung rein digitaler Services (beispielsweise die rein digital abgewickelte Beratung zu Tourismusangeboten) und zielen auf den Consumer-Bereich.

\section{Frühzeitig über den Einsatz von Crowd Services entscheiden}

Die Anzahl der Unternehmen, die Crowdworking-Plattformen nutzt, steigt. Das Prinzip der Erbringung von über Crowdworking-Plattformen beauftragten Services ,von Kunden für Kunden" scheint auf Grund der oben skizzierten Vorteile aktuell auch im deutschsprachigen Raum Zulauf zu bekommen: So hat am 17. Januar 2017 die Plattform „iAdvize“ mit Hauptsitz im französischen Nantes den Launch ihrer neuen Service-Plattform ,ibbü“ (www.ibbu.com/de) für Deutschland bekannt gegeben, die Crowd Services für verschiedene Branchen vermittelt. Hält dieser Trend an und wollen immer mehr Unternehmen solche Crowd Services nutzen, dann kommt es nach Einschätzung der Autoren dieses Beitrages mittelfristig zu einer Knappheit an verfügbaren Crowd Workern. Es gilt daher für Unternehmen, frühzeitig über den Einsatz von Crowd Services zu entscheiden. Bei einer Entscheidung für einen solchen Einsatz kann eine First-Mover-Advantage gegenüber Mitwettbewerbern erzielt sowie frühzeitig eine eigene Crowd aufgebaut und an sich gebunden werden.

\section{Neue Kundenschnittstellen in die bisherigen Kanäle integrieren}

Ist die Entscheidung gefallen, Crowd Services zu nutzen, gilt es, diese im Sinne einer effizienten Multikanalstrategie in die Gesamtheit der Vertriebskanäle des Unternehmens wie Apps, Call Center, Internet-Auftritt, Ladengeschäfte, u.v.m., sinnvoll zu integrieren. Für das Beispiel Swisscom haben wir in den obigen Abschnitten gezeigt, dass durch die Nutzung der Crowdworking-Plattform Mila weitere digitale Kundenschnittstellen entstehen und sind auf die daraus resultierenden Vorteile eingegangen. Ein Grund für das erfolgreiche Zusammenspiel von Mila und Swisscom ist, dass je nach Bedarf verschiedene Kanäle und Beauftragungsmöglichkeiten für Services verfügbar sind. So besteht beispielsweise auf dem Internet-Auftritt für Privatkunden von Swisscom die Möglichkeit, per Link direkt auf den Internet-Auftritt von Mila zu gehen und dort dann Services über Swisscom Friends zu beauftragen (siehe Abbildung 4). Eine weitere digitale Kundenschnittstelle resultiert aus der neuen Mila Mobile App (siehe Abbildung 3). Eine enge Integration der verschiedenen (,,analogen“ und digitalen) Kundenschnittstellen hat zudem das Potenzial, den erwähnten Nachteil im Bereich „Corporate Identity“, der aus einem zum Kunden hin nicht als einheitlich wahrgenommenen Auftritt resultiert, so weit wie möglich zu begrenzen. 


\section{Externe mit internen Crowds (eigenen Mitarbeitern) eng verzahnen}

Bei der Darstellung der Nachteile haben wir deutlich gemacht, dass durch den Einsatz externer Crowds in bereits bestehenden Vertriebsgebieten eine Verunsicherung der internen Mitarbeiter bis hin zu Jobverlustängsten stattfinden kann. Durch eine enge Verzahnung der externen Crowd Worker mit den internen Mitarbeitern eines Unternehmens können solche Verunsicherungen reduziert oder sogar weitgehend vermieden werden (Mrass et al. 2017a). Insbesondere wenn deutlich wird, dass diese Crowd schwerpunktmäßig komplementäre Services bietet und bestehende Servicelücken im Angebot des Unternehmens schließt. Unternehmen sollten also Möglichkeiten einer engen Verzahnung externer und interner Crowds prüfen.

\section{Kontinuierlich in die Motivation der Crowd investieren}

Die Erkenntnisse der Autoren dieses Beitrages im Rahmen ihrer allgemeinen Forschungs zu Crowdworking-Plattformen, unter anderem aus ein- bis zweistündigen Tiefen-Interviews mit CEO's und Geschäftsführern von insgesamt 14 CrowdworkingPlattformen mit Sitz oder einem physischen Standort in Deutschland (siehe Tabelle 1), zeigen eine große gemeinsame Herausforderung: Eine engagierte, gut qualifizierte und hoch motivierte Crowd kontinuierlich zu gewährleisten und diese langfristig an sich zu binden. Ebenso wie Unternehmen Crowd Worker „on demand“ beauftragen können und für sie dabei im Gegensatz zu internen Mitarbeitern keine Fixkosten verbunden sind, können sich umgekehrt auch Crowd Worker ohne allzu große Wechselkosten (vom Verlust der auf einer Crowdworking-Plattform gewonnenen Reputation abgesehen) einer anderen Plattform beziehungsweise auf der gleichen Plattform den Aufträgen anderer Unternehmen zuwenden. Erkenntnisse der Autoren dieses Beitrages aus ihrer allgemeinen Forschung zu Crowdworking-Plattformen zeigen zudem, dass eine große Anzahl an Crowd Workern auf den jeweiligen Plattformen inaktiv ist (und unterstreichen damit die Bedeutung der kontinuierlichen Motivation der Crowd). Maßnahmen wie Prämien für besonders gute Beratungen, Reputationsmechanismen wie beispielsweise SterneKlassifizierungssysteme, die vorzeitige Bereitstellung neuer Produkte vor Markteinführung zu Testzwecken oder Umsatzbeteiligungen können aus unserer Sicht helfen, die Loyalität der Crowd und ihre Motivation für die Erbringung von Services für das jeweilige Unternehmen zu erhalten oder zu steigern.

\section{Die Crowd für eine Erweiterung des Vertriebsgebietes nutzen}

Anhand des Beispiels Mila lässt sich zeigen, dass Unternehmen das eigene Vertriebsgebiet durch den Einsatz von Crowd Services schnell und kostengünstig erweitern können. Und dass Unternehmen damit auch in Regionen, in denen sie bisher nicht oder nur wenig präsent sind, mittels der Crowd Services erbringen lassen und den Vertrieb ihrer Produkte auch außerhalb des bisherigen Geschäftsgebietes unterstützen können. Insbesondere wenn die Strategie eines Unternehmens eine Expansion in kurzer Zeit vorsieht, sind Crowd Services damit eine kostengünstige und schnell implementierbare Alternative zum oft langwierigen und mühsamen Aufbau eines eigenen internen Vertriebs. Vor allem auch um erst einmal zu testen, wie die Produkte und Services in neuen potenziellen Vertriebsregionen ankommen, ist ein schneller Rollout von Services mittels des Einsatzes von Crowds gut geeignet. Gleichzeitig kann ebenso bei Bedarf ein zeitnaher „Rollback“ erfolgen, wenn die Erfahrungen darauf hindeuten, dass die jeweilige Region für die eigenen Produkte und Services nicht Erfolg versprechend ist.

In diesem Beitrag haben wir anhand des Beispiels der Crowdworking-Plattform Mila und ihres Kunden Swisscom gezeigt, dass Crowd Services der Art „Von Kunden, für Kunden“ eine gute Ergänzung und Erweiterung des bestehenden Service-Angebotes eines Unternehmens und 
damit seiner Digital Customer Experience darstellen können. Sie liegen im Trend der On-Demand- und der Sharing-Economy und tragen der Tatsache Rechnung, dass Kunden einerseits immer öfter zu Produzenten von Services werden und andererseits bedarfsgerechte Services „,auf Knopfdruck“ erwarten. Crowd Services bieten zudem Chancen, den aus abnehmender Kundenloyalität und schnellen Wechseln zu anderen Anbietern entstehenden Herausforderungen zu begegnen. Gleichwohl es Maßnahmen bedarf, um potenziellen Nachteilen entgegenzuwirken, können aus dem Einsatz von Crowd Services erhebliche Vorteile in den Bereichen Effizienz, Geschwindigkeit, Kosten, Reichweite und Glaubwürdigkeit resultieren. Das Beispiel Mila (die durchschnittliche Bewertung beträgt nach Unternehmensangaben 4,8 von 5 möglichen Punkten und 92 Prozent der Kunden würden wieder beauftragen) zeigt zudem, dass die Zufriedenheit der Kunden auch bei Einsatz solcher Crowd Services sehr hoch sein kann.

Das Prinzip, über Crowdworking-Plattformen beauftragte Services ,,von Kunden für Kunden" erbringen zu lassen, bekommt wie das Beispiel iAdvize mit dem Launch der neuen Service-Plattform ibbü (www.ibbu.com/de) zeigt, auch in Deutschland weiter Zulauf. Das Auftreten dieser neuen Art von Crowdworking-Plattformen für Crowd Services ist ein weiteres Indiz dafür, dass es sich hierbei um ein attraktives Instrument handelt. Und dass früher oder später viele Unternehmen dieses zur Ergänzung und Erweiterung ihrer Services nutzen könnten. Ziel könnte dabei sein, Wettbewerbsvorteile zu generieren und gegenüber Konkurrenten nicht zurückzufallen. Unternehmen, die diese Entwicklung bereits frühzeitig antizipieren, profitieren angesichts der Erwartung, dass die Anzahl an verfügbaren Crowd Workern für solche Services mittelfristig begrenzt sein wird, von einer First-Mover-Advantage. Auch wenn die durch Kunden erbrachten Services hinsichtlich deren Qualität nicht immer mit denjenigen von professionellen eigenen Beschäftigten vergleichbar sind und komplexere Arbeit meist noch internen Mitarbeitern vorbehalten ist, sind Kunden, die das Produkt des jeweiligen Unternehmens tatsächlich auch selbst nutzen, gute Markenbotschafter. Es erhöht die Glaubwürdigkeit bei der Beratung und Erbringung von Dienstleistungen, wenn sich jemand bereits selbst privat für das jeweilige Produkt entschieden hat und daher sowohl dessen Stärken als auch eventuelle Schwächen gut kennt. Crowd Services zu nutzen bietet für Unternehmen nicht zuletzt die Möglichkeit, ohne den kostspieligen Aufbau eines eigenen Kundendienstes und Vertriebes auch in Regionen, die bisher nicht oder nur schwach abgedeckt wurden, eine Präsenz aufzubauen. Weitere Forschung in diesem Themengebiet sollte sich insbesondere damit beschäftigen, wie Anreiz- und Motivationsmechanismen gestaltet werden können, die Entwicklungskonzepte im Bereich Smart City unterstützen und insbesondere der Tatsache Rechnung tragen, dass in Zukunft die Nachfrage nach Crowd-Lösungen für die Bearbeitung komplexer Arbeit weiter ansteigen wird.

Die obigen fünf Punkte stellen erste wichtige konkrete Handlungsempfehlungen für Unternehmen dar, die den Wert ihrer digitalen Schnittstellen zum Kunden erhöhen und aus der Nutzung von Crowd Services mögliche Wettbewerbsvorteile generieren wollen. CrowdworkingPlattformen für die Erbringung von Beratungs- und Vertriebsleistungen ,,von Kunden für Kunden" bieten erhebliches Potenzial, das für verschiedene Arten von Arbeit innerhalb recht heterogener Branchen genutzt werden kann. Unternehmen, die potenziell von dem Einsatz solcher Crowd Services profitieren können und sich diesem Trend zu lange verschließen, riskieren nach Überzeugung der Autoren dieses Beitrages im Wettbewerb langfristig zurückzufallen.

\section{Danksagung}

Dieser Beitrag entstand im Rahmen des seitens des Bundesministeriums für Bildung und Forschung (BMBF) geförderten Projektes „Herausforderung Cloud und Crowd - Neue Organisationskonzepte für Dienstleistungen nachhaltig gestalten“ (Förderkennzeichen: 02K14A071, Projektträger: PTKA/Projektträger Karlsruhe am Karlsruher Institut für Technologie/KIT). 


\section{Literatur}

Alter S (2013) Work System Theory: Overview of Core Concepts, Extensions, and Challenges for the Future. Journal of the Association for Information Systems (JAIS) 14:72-121

Brynjolfsson E, McAfee A (2011) Race against the machine; How the digital revolution is accelerating innovation, driving productivity, and irreversibly transforming employment and the economy. Digital Frontier Press, Lexington, Mass.

Kollmann T, Schmidt H (2016) Deutschland 4.0; Wie die Digitale Transformation gelingt. Springer Gabler, Wiesbaden

Leimeister JM, Zogaj S (2013) Neue Arbeitsorganisation durch Crowdsourcing; Eine Literaturstudie

Mrass V, Peters C (2017) Crowdworking-Plattformen in Deutschland. University of Kassel

Mrass V, Peters C, Leimeister JM (2016) New Work Organization through Crowdworking Platforms; A Case Study. Konferenz "Zukunftsprojekt Arbeitswelt 4.0", 19.09.2016, Stuttgart

Mrass V, Peters C, Leimeister JM (2017a) One for All? Managing External and Internal Crowds through a Single Platform - A Case Study. 50th Hawaii International Conference on System Sciences (HICSS), 2017:4324-4333

Mrass V, Li MM, Peters C (2017b) Towards a Taxonomy of Digital Work. 25. European Conference on Information Systems (ECIS), Guimarães, Portugal, 2017

Surowiecki J (2004) The wisdom of crowds; Why the many are smarter than the few and how collective wisdom shapes business, economies, societies, and nations. Doubleday, New York

Yin RK (2014) Case Study Research; Design and Methods. SAGE Publications, Inc., Los Angeles 